\title{
Multi-cell Interference Coordination for Multigroup Multicast Transmission
}

\author{
Oskari Tervo*, Harri Pennanen*, Symeon Chatzinotas ${ }^{\ddagger}$, Björn Ottersten ${ }^{\ddagger}$, and Markku Juntti* \\ ${ }^{*}$ Centre for Wireless Communications, University of Oulu, Oulu, Finland \\ ${ }^{\ddagger}$ the Interdisciplinary Centre for Security, Reliability and Trust, University of Luxembourg, Luxembourg \\ Email: \{firstname.lastname\}@oulu.fi, \{symeon.chatzinotas, bjorn.ottersten\}@uni.lu
}

\begin{abstract}
Multicasting has become a particularly important technique in the context of cache-enabled cloud radio access networks proposed for $5 \mathrm{G}$ systems, where it can be used to transmit common information to multiple users to improve both spectral and energy efficiency. For the efficient spectrum utilization, the future communications are based on aggressive frequency reuse, where the required data rates can be achieved with multiple-input multiple-output precoding techniques. This approach, however, calls for advanced interference coordination techniques. This paper summarizes some of the core approaches proposed in the literature and discusses the main future challenges.
\end{abstract}

Index Terms-Coordinated beamforming, multicasting, cloud radio access network, caching.

\section{INTRODUCTION}

The upcoming years are expected to bring 45 percent yearly increase in the global mobile data traffic, resulting in a tenfold increase between 2016 and 2022 [1], [2]. The rapid evolution of wireless communications such as the increasing popularity of the smart phones is causing significant changes on the type of communications. The shift toward content-centric transmission like video streaming or mobile television means that more and more users want to access the same information at the same time. To overcome this challenge, multicasting has received an increasing attention in the literature. The cloud radio access network architecture (CRAN) which has been proposed to respond to the huge future data traffic and latency requirements enables multi-cell cooperation and flexible platform for efficient communications. In particular, the multicasting transmission combined with caching of the popular contents enables high data rate applications with good spectral and energy efficiency [3], [4]. Moreover, for the efficient spectrum utilization, the future communications are based on aggressive frequency reuse, where the required data rates can be achieved with multiple-input multiple-output (MIMO) precoding techniques. Although MIMO precoding together with CRAN architecture has a huge potential, it poses many challenges in terms of smart cooperation, user allocation/scheduling, and interference coordination. The interference between the different data streams has to be addressed via advanced interference coordination methods when designing the precoding.
The core difference of multicasting from the conventional unicasting transmission is that a common beamformer is designed for a group of users, which means that the transmitted common data rate for the group is determined by the user having the worst channel conditions. This makes even the conventional transmit power minimization problem NP-hard [5], [6]. Multicast beamforming problems have been studied in single-cell systems for different optimization targets, e.g., transmit power minimization [5], [6], signal-to-interferenceplus noise ratio (SINR) balancing [6], [7], and sum rate maximization [8]. Coordinated multicast single group beamforming for transmit power minimization and max-min fairness has been studied in [9], where the semidefinite relaxation (SDR) was used to solve these problems. Bornhorst et al. [10] proposed a distributed successive convex approximation (SCA) based method to solve the multicasting problem in the relay networks with single-antenna nodes. The weighted SINR balancing problem with more practical per-antenna power constraints was considered in [7], [11], and the work was extended to the sum rate maximization problem in [12]. He et al. [13] proposed a centralized algorithm for the energy efficiency maximization problem in multi-cell multigroup system. One of the important limitations in the multi-antenna beamforming is that the number of radio frequency (RF) chains is limited due to complexity and cost issues. This limitation was taken into account in [14], where the problems of joint beamforming and antenna selection for transmit power minimization and maxmin fairness were studied. The antenna selection problem was considered as a sparse beamforming solution, which resulted in semidefinite program (SDP) together with semidefinite relaxations. From the energy efficiency viewpoint, the joint beamforming and antenna selection problem was recently studied in [15], where the continuous relaxation together with successive convex approximation algorithm was applied to solve the problem.

In this paper, we briefly summarize the coordinated multicast beamforming techniques proposed in the literature and discuss the motivation and challenges of multicasting in future wireless communications. Section II presents the system model and discusses the differences between unicasting and multicasting. The overview of the methods is provided in Section III, Section IV provides numerical examples and future challenges 
are discussed in $\mathrm{V}$.

\section{System Model And PRoblem Formulation}

\section{A. System Model}

A generic multi-cell multigroup multicasting system is illustrated in Fig. 1. It includes $B$ BSs, where each BS $b \in \mathcal{B}=\{1, \ldots, B\}$, transmits information to $G$ groups of users in the coverage area. Each group receives different independent information but the message for the users within each group is common. The set of groups served by BS $b$ is denoted by $\mathcal{G}_{b}=\left\{j, j+1, \ldots, j+G_{b}-1\right\}$, where $G_{b}$ is the number of groups served by $\mathrm{BS} b$, and $j$ is a group index. The total number of users and groups in the network is denoted by $K=|\mathcal{K}|(\mathcal{K}=\{1, \ldots, K\})$ and $G=|\mathcal{G}|(\mathcal{G}=\{1, \ldots, G\})$, respectively. BS $b$ is equipped with $N_{b}$ transmit antennas, whereas each user has only one receive antenna. The serving BS of user group $g$ is denoted as $b_{g}$. The set of users in group $g$ is denoted by $\mathcal{K}_{g} \subset \mathcal{K}$. Since each user belongs to only one group, the sets of users belonging to different groups are disjoint, i.e., $\mathcal{K}_{i} \cap \mathcal{K}_{j}=\emptyset, \forall i, j \in \mathcal{G}, i \neq j$. The received signal at user $k$ in group $g$ is given by

$$
\begin{aligned}
y_{k}= & \overbrace{\mathbf{h}_{b_{g}, k}^{H} \mathbf{w}_{g} s_{g}}^{\text {desired signal }}+\overbrace{\sum_{i \in \mathcal{G}_{b} \backslash\{g\}} \mathbf{h}_{b_{g}, k}^{H} \mathbf{w}_{i} s_{i}}^{\text {intra-cell interference }} \\
& +\underbrace{\sum_{j \in \mathcal{B} \backslash\left\{b_{g}\right\}} \sum_{u \in \mathcal{G}_{j}} \mathbf{h}_{j, k}^{H} \mathbf{w}_{u} s_{u}}_{\text {inter-cell interference }}+n_{k}
\end{aligned}
$$

where $\mathbf{h}_{b, k} \in \mathcal{C}^{N_{b} \times 1}$ is the channel vector from BS $b$ to user $k, \mathbf{w}_{g} \in \mathcal{C}^{N_{b} \times 1}$ is the transmit beamforming vector of group $g, s_{g} \in \mathcal{C}$ is the corresponding normalized data symbol and $n_{k} \sim \mathcal{C N}\left(0, \sigma_{k}^{2}\right)$ is the complex white Gaussian noise sample with zero mean and variance $\sigma_{k}^{2}$. The SINR of user $k$ can be written as

$$
\Gamma_{k}(\mathbf{w}) \triangleq \frac{\left|\mathbf{h}_{b_{g}, k}^{H} \mathbf{w}_{g}\right|^{2}}{\sigma_{k}^{2}+\sum_{u \in \mathcal{G} \backslash\{g\}}\left|\mathbf{h}_{b_{u}, k}^{H} \mathbf{w}_{u}\right|^{2}}
$$

Since the same information is transmitted to multiple users in a group, the transmission rate is determined by the user having the worst channel condition in the group. As a result, the data rate for user group $g$ is given as

$$
R_{g}(\mathbf{w}) \triangleq \min _{k \in \mathcal{K}_{g}} \log \left(1+\Gamma_{k}(\mathbf{w})\right)
$$

\section{B. From Unicasting to Multicasting}

The previous cellular network standards have been designed to unicasting operations [4]. The reason for increasing interest of multicasting is the nature of future communications. The development of smart devices enables, e.g., online video streaming everywhere and a number of users may request the same data at the same time. In order to use scarce frequency spectrum in an efficient manner, multicasting can significantly save transmission resources with improved QoS compared to the unicasting case where the same information

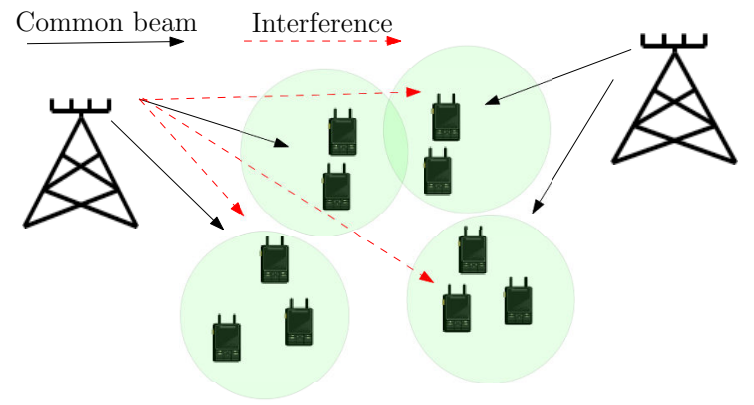

Fig. 1. Multi-cell multigroup multicasting system.

would be transmitted in different resources [16]. This is also recognized in LTE specifications by the name Evolved Multimedia Broadcast and Multicast Services (eMBMS) [17]. Obviously, the benefits of multicasting over unicasting depend on the user distribution. For example, when the population is concentrated in a small geographical area, multicasting is an appropriate way of delivering the same requested data to large number of users. However, if the user distribution is more sparse, unicasting may provide better alternative. The 3GPP standards and the commercial versions of eMBMS, e.g., LTE broadcast presented by Qualcomm [18], enable dynamic switching between unicasting and multicasting, which improves the scalability and flexibility of multicasting.

In the conventional unicasting transmission [19]-[21], each user receives independent information from one or more BSs. This means that the BSs are able to construct independent narrow beams toward each user. However, in a multicasting transmission, the BSs can only design a common wider beam to a user group receiving the common information. In particular, a beamformer designed for one group impacts not only to the desired signal power of one user, but to the signal power of all the users in one group. As a result, even the conventional power minimization problem [19] becomes more difficult to solve. Specifically, the traditional optimal approach from unicasting which converts the power minimization problem to an SOCP [19] is no longer applicable. Another approach based on the standard SDR which converts the same problem to SDP cannot necessarily solve the multicasting power minimization problem optimally because the unit rank solution cannot be guaranteed. However, many suboptimal approaches have been already provided in the literature, and we briefly review a few of them for different optimization metrics in Section III.

\section{INTERFERENCE COORDINATION FOR MULTICASTING}

\section{A. Sum Power Minimization}

Due to the aggressive frequency reuse, the main problem in multi-cell systems is to address the resulting inter-cell interference. For the power minimization target, this problem was solved in [9], [22], where both centralized and distributed solutions have been proposed. More specifically, the optimization target is to minimize the total transmit power with userspecific QoS constraints. This problem can be cast as 


$$
\begin{array}{ll}
\underset{\left\{\mathbf{w}_{g}\right\}_{g \in \mathcal{G}}}{\min } & \sum_{g \in \mathcal{G}} \operatorname{Tr}\left(\mathbf{w}_{g} \mathbf{w}_{g}^{H}\right) \\
\text { s. t. } & \frac{\left|\mathbf{h}_{b_{g}, k}^{H} \mathbf{w}_{g}\right|^{2}}{\sigma_{k}^{2}+\sum_{j \in \mathcal{B}} \sum_{u \in \mathcal{G}_{j} \backslash\{g\}}\left|\mathbf{h}_{j, k}^{H} \mathbf{w}_{u}\right|^{2}} \geq \gamma_{k} \\
& \forall b \in \mathcal{B}, \forall g \in \mathcal{G}_{b}, \forall k \in \mathcal{U}_{g}
\end{array}
$$

where $\gamma_{k}$ is the minimum SINR requirement for user $k$. The SINR requirement can be equivalently mapped to a minimum rate requirement. Problem (4), which is a more generic multicell version of the problem in [5], is also non-convex and NP-hard, and thus, cannot be solved in its current form. The standard SDR can be applied to approximate the problem as

$$
\begin{array}{ll}
\underset{\left\{\mathbf{W}_{g}\right\}_{g \in \mathcal{G}}}{\min } & \sum_{g \in \mathcal{G}} \operatorname{Tr}\left(\mathbf{W}_{g}\right) \\
\text { s. t. } & \frac{\operatorname{Tr}\left(\mathbf{H}_{b_{g}, k} \mathbf{W}_{g}\right)}{\sigma_{k}^{2}+\sum_{j \in \mathcal{B}} \sum_{u \in \mathcal{G}_{j} \backslash\{g\}} \operatorname{Tr}\left(\mathbf{H}_{j, k} \mathbf{W}_{u}\right)} \geq \gamma_{k} \\
& \forall b \in \mathcal{B}, \forall g \in \mathcal{G}_{b}, \forall k \in \mathcal{U}_{g} \\
& \mathbf{W}_{g} \succeq 0, \forall g \in \mathcal{G}
\end{array}
$$

which is a convex SDP. Although the above problem can be solved optimally, the optimality of the original problem (4) is not guaranteed because the optimal covariance matrices are not necessarily rank-1. To this end, Gaussian randomization has been used to find feasible beamforming solution from the covariance matrices. Problem (5) can be efficiently solved via centralized processing, where each base station has to share the local channel information to a central controller. However, the signaling overhead of the centralized approach scales with the number of antennas, and it also incurs additional delays. To reduce the signaling overhead and be more robust in the time-varying channels, [9], [22] have also proposed distributed methods based on primal decomposition. The distributed approaches only require sharing the user-specific intercell interference variables, i.e., the signaling overhead only scales with the number of users. The distributed solutions can provide more practical approach especially with high number of antennas because sharing all the channel information to a central controller or baseband unit in the CRAN systems may be difficult to realize with reasonable delays.

An alternative solution was proposed in [23] by explicitly replacing the SINR constraints with rate constraints, i.e., $\log \left(1+\Gamma_{k}(\mathbf{w})\right) \geq \bar{R}_{k}$. The authors proposed successive convex approximation based methods both with and without applying the SDR.

\section{B. SINR Balancing}

The problem of fair minimum SINR maximization in multiuser MISO systems with more practical per-antenna power constraints was studied in [7]. This problem can be cast as

$$
\begin{aligned}
& \underset{\left\{\mathbf{w}_{g}\right\}_{g \in \mathcal{G}}}{\max } \min _{k \in \mathcal{K}} \frac{1}{\omega_{k}} \frac{\left|\mathbf{h}_{b_{g}, k}^{H} \mathbf{w}_{g}\right|^{2}}{\sigma_{k}^{2}+\sum_{j \in \mathcal{B}} \sum_{u \in \mathcal{G}_{j} \backslash\{g\}}\left|\mathbf{h}_{j, k}^{H} \mathbf{w}_{u}\right|^{2}} \\
& \text { s. t. } \quad\left[\sum_{g \in \mathcal{G}_{b}} \mathbf{w}_{g} \mathbf{w}_{g}^{H}\right]_{n n} \leq P_{b, n}, \forall b \in \mathcal{B}, n \in \mathcal{N}_{b}
\end{aligned}
$$

where $\omega_{k} \geq 1$ is some priority weight for user $k$. The above problem can be equivalently expressed as

$$
\begin{aligned}
& \max . \quad t \\
& t,\left\{\mathbf{w}_{g}\right\}_{g \in \mathcal{G}} \\
& \text { s. t. } \frac{1}{\omega_{k}} \frac{\left|\mathbf{h}_{b_{g}, k}^{H} \mathbf{w}_{g}\right|^{2}}{\sigma_{k}^{2}+\sum_{j \in \mathcal{B}} \sum_{u \in \mathcal{G}_{j} \backslash\{g\}}\left|\mathbf{h}_{j, k}^{H} \mathbf{w}_{u}\right|^{2}} \geq t, \forall k \in \mathcal{K} \\
& {\left[\sum_{g \in \mathcal{G}_{b}} \mathbf{w}_{g} \mathbf{w}_{g}^{H}\right]_{n n} \leq P_{b, n}, \forall b \in \mathcal{B}, n \in \mathcal{N}_{b}}
\end{aligned}
$$

where $t$ is a new variable representing the minimum SINR which has to be satisfied by all the users in the network. Again, by using the standard SDR, the above non-convex problem can be transformed as

$$
\begin{aligned}
& \operatorname{max.}_{t,\left\{\mathbf{W}_{g}\right\}_{g \in \mathcal{G}}} t \\
& \text { s. t. } \frac{1}{\omega_{k}} \frac{\operatorname{Tr}\left(\mathbf{H}_{b_{g}, k} \mathbf{W}_{g}\right)}{\sigma_{k}^{2}+\sum_{j \in \mathcal{B}} \sum_{u \in \mathcal{G}_{j} \backslash\{g\}} \operatorname{Tr}\left(\mathbf{H}_{j, k}^{H} \mathbf{W}_{u}\right)} \geq t, \forall k \in \mathcal{K} \\
& {\left[\sum_{g \in \mathcal{G}_{b}} \mathbf{W}_{g}\right]_{n n} \leq P_{b, n}, \forall b \in \mathcal{B}, n \in \mathcal{N}_{b}}
\end{aligned}
$$

For fixed $t,(8)$ is convex and, thus, can be solved via bisection method in the interval $t_{\min }$ and $t_{\max }$. After finding the optimal covariance matrices, the Gaussian randomization can be used to find feasible beamformers if the solution is not rank-1.

\section{Sum Rate Maximization}

The sum rate maximization for single-cell multigroup multicasting was considered in [12]. To express the problem in a multi-cell system, we can write

$$
\begin{array}{ll}
\underset{\left\{\mathbf{w}_{g}\right\}_{g \in \mathcal{G}}}{\max } & \sum_{g \in \mathcal{G}} \min _{k \in \mathcal{K}_{g}} \log \left(1+\Gamma_{k}(\mathbf{w})\right) \\
\text { s. t. } & {\left[\sum_{g \in \mathcal{G}_{b}} \mathbf{w}_{g} \mathbf{w}_{g}^{H}\right]_{n n} \leq P_{b, n}, \forall b \in \mathcal{B}, n \in \mathcal{N}_{b}}
\end{array}
$$

Christopoulos et al. [12] proposed a heuristic iterative algorithm to solve this problem. Firstly, the beamforming directions $\tilde{\mathbf{w}}$ were solved to minimize the per-antenna minimization problem with some SINR targets. Subsequently, the minimum group-specific SINR targets are calculated as the minimum SINR per group $\gamma_{g}(\tilde{\mathbf{w}})=\min _{k \in \mathcal{K}_{g}}\left(\Gamma_{k}(\tilde{\mathbf{w}})\right)$. Then, by fixing the target SINR values, the power allocation was solved via subgradient method. The intuition behind the method is that any additional power which is still available after solving the per-antenna power minimization problem, is used to increase the total sum rate.

\section{Energy Efficiency Maximization with Antenna Selection}

The work of [15] studied energy efficiency maximization by jointly optimizing multicast beamformers and active antennas. The optimization target is to maximize network energy efficiency

$$
\begin{array}{cl}
\max _{\mathbf{w}, \mathbf{a}} & \frac{\sum_{g \in \mathcal{G}} \min _{k \in \mathcal{K}_{g}} \log \left(1+\Gamma_{k}(\mathbf{w})\right)}{\sum_{g \in \mathcal{G}} \frac{1}{\eta}\left\|\mathbf{w}_{g}\right\|_{2}^{2}+P_{\mathrm{RF}} \sum_{b \in \mathcal{B}} \sum_{i \in \mathcal{N}_{b}} a_{b, i}+P_{\text {sta }}} \\
\text { s.t. } & \left\|\hat{\mathbf{w}}_{b, i}\right\|_{2}^{2} \leq a_{b, i} P_{\max }, \forall b \in \mathcal{B}, i \in \mathcal{N}_{b} \\
& \Gamma_{k}(\mathbf{w}) \geq \gamma_{k}, \forall k \in \mathcal{K},
\end{array}
$$




$$
a_{b, i} \in\{0,1\}, \forall b \in \mathcal{B}, i \in \mathcal{N}_{b}
$$

where $\eta \leq 1$ is the power amplifier efficiency which is assumed to be fixed for simplicity, $P_{\mathrm{RF}}$ is power consumption per active RF chain, $P_{\text {sta }}$ is a fixed power consumption value consumed for signal processing and other related operations, $\mathbf{w} \triangleq\left\{\mathbf{w}_{g}\right\}_{g \in \mathcal{G}}, \mathbf{a} \triangleq\left\{a_{b, i}\right\}_{b \in \mathcal{B}, i \in \mathcal{N}_{b}}, \hat{\mathbf{w}}_{b, i} \triangleq$ $\left[\mathbf{w}_{j}[i], \mathbf{w}_{j+1}[i], \ldots, \mathbf{w}_{j+G_{b}-1}[i]\right]$ includes the beamforming coefficients related to antenna $i$ of $\mathrm{BS} b$ and index $j$ refers to a group index served by BS $b$. The constraint in (10b) guarantees that if $a_{b, i}=0$, then the beamformers associated with antenna $i$ are set to zero. Due to the binary variables a, the above problem is categorized as mixed-Boolean non-linear program which is difficult to solve. First, the new equivalent transformation was proposed by replacing (10b) with

$$
\begin{aligned}
& \left\|\hat{\mathbf{w}}_{b, i}\right\|_{2}^{2} \leq a_{b, i}^{\alpha} v_{b, i}, \forall b \in \mathcal{B}, i \in \mathcal{N}_{b} \\
& v_{b, i} \leq P_{\max }, \forall b \in \mathcal{B}, i \in \mathcal{N}_{b}
\end{aligned}
$$

where $v_{b, i}$ is a new variable representing the allocated power for antenna $(b, i), \alpha \geq 1$ is a fixed penalty parameter which reduces the feasible set of the problem, and potentially yields close to binary solutions when the continuous relaxation is applied. We proposed a successive convex approximation based algorithm [15], where the binary variables are relaxed to take continuous values in the interval $[0,1]$. In particular, the following problem is iteratively solved

$$
\begin{aligned}
\max _{\mathbf{w}, \boldsymbol{\gamma}, \mathbf{v}, \mathbf{a}, \boldsymbol{\beta}, \mathbf{r}} & \frac{\sum_{g \in \mathcal{G}} r_{g}}{\sum_{b \in \mathcal{B}} \sum_{i \in \mathcal{N}_{b}} \frac{1}{\eta} v_{b, i}+P_{\mathrm{RF}} \sum_{b \in \mathcal{B}} \sum_{i \in \mathcal{N}_{b}} a_{b, i}+P_{\text {sta }}}(12 \mathrm{a}) \\
\text { s.t. } & \frac{\left\|\hat{\mathbf{w}}_{b, i}\right\|_{2}^{2}}{v_{b, i}} \leq \chi_{b, i}^{(n)}\left(a_{b, i}\right), \forall b \in \mathcal{B}, i \in \mathcal{N}_{b} \\
& \gamma_{k} \leq \Psi_{k}^{(n)}\left(\mathbf{w}_{g}, \beta_{k}\right), \forall k \in \mathcal{K} \\
& 0 \leq a_{b, i} \leq 1, \forall b \in \mathcal{B}, i \in \mathcal{N}_{b} \\
& \text { other convex constraints. }
\end{aligned}
$$

by updating the linear approximations $\chi_{b, i}^{(n)}\left(a_{b, i}\right)$ and $\Psi_{k}^{(n)}\left(\mathbf{w}_{g}, \beta_{k}\right)$ after each iteration according to the SCA principle [24]. The problem is a concave-convex fractional program which can be solved by using the Charnes-Cooper transformation [25]. Although the antenna selection variables are continuous, they are illustrated to converge close to binary variables due to the new formulation (12b).

\section{NumericAl RESUltS}

We evaluate the performance for a quasistatic frequency flat Rayleigh fading channel model with $B=2$ base stations. Each BS serves two groups of users with $\left|\mathcal{K}_{g}\right|=L$ users per group. The considered system model is a worst case interference system where all the user groups are co-located so that the average path loss from all the BSs to all the users is $0 \mathrm{~dB}$. We assume a unit bandwidth and noise power is normalized to $N_{0}=0 \mathrm{dBW}$. We set $N_{b}=N=12, \forall b \in \mathcal{B}$, and $\gamma_{k}=\gamma, \forall k \in \mathcal{K}$.

Due to the fact that the common beamformer is designed for many users in the multicasting transmission, it is obvious

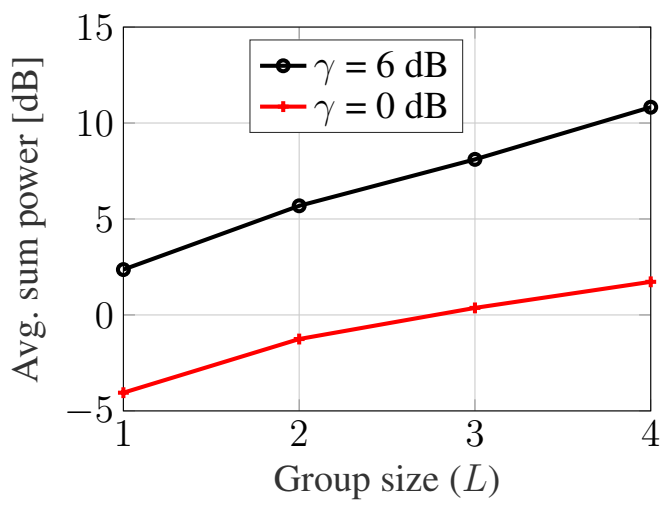

Fig. 2. The effect of multicasting group size on the required minimum transmit power.

that the performance decreases when the user group size increases. The reason for this is that the transmitted beams are wider due to the user separation, which simply means that more power is required to achieve certain QoS thresholds and the resulting interference increases. This phenomena is illustrated in Fig. 2, where the minimum total transmit power is plotted against the group size. In particular, the number of transmitted data streams remains fixed but more users request each of the streams. It is observed that the power consumption approximately doubles when the group size doubles. The increase in the average interference power is illustrated in Fig. 3. We can see that there is a significant increase in the optimal interference level which shows the importance of interference coordination. Interestingly, for the case of unicasting (group size 1), the higher SINR levels are achieved by allowing lower interference level.

In the final example, we compare the performance of multicasting and unicasting. Specifically, we have solved the power minimization problem with the multicasting strategy, where each group contains $L$ users as shown in x-axis, and compared it with the method where each user in each multicasting group is served in a unicasting manner with orthogonal resources. All the users have got exactly the same average service level and equal number of resources are consumed as in the multicasting case. It is observed that multicasting strategy achieves significantly superior performance to unicasting, demonstrating the better spectrum efficiency. We can also see that the benefits of multicasting increase with higher QoS levels.

\section{Future Challenges}

Multicasting is an efficient technology for popular content delivery. However, the intelligent use of it is not a trivial task. For example, the decision whether the specific content within a specific location is popular enough should be done smartly, since it takes resources from unicasting traffic. This calls for wise user allocation and scheduling of unicasting/multicasting users. Another important issue requiring careful planning is the joint design of user/BS clustering and beamforming since the BSs are densely deployed in future cellular systems. The MIMO techniques are also limited by the number of RF chains, for which advanced antenna selection strategies can 


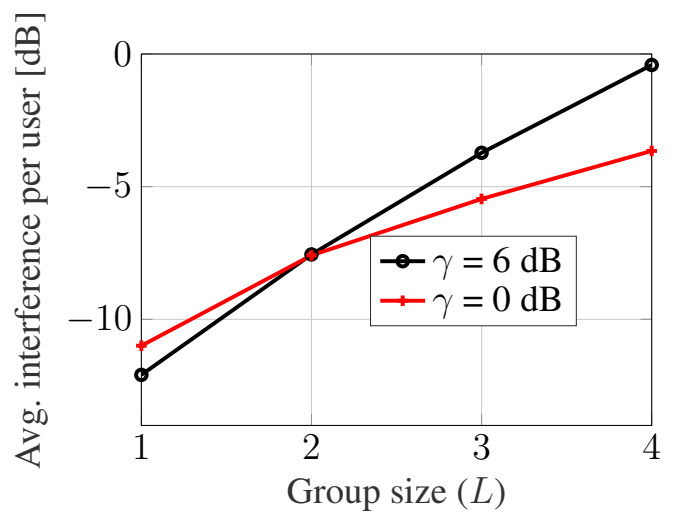

Fig. 3. The effect of multicasting group size on the average interference power per user.

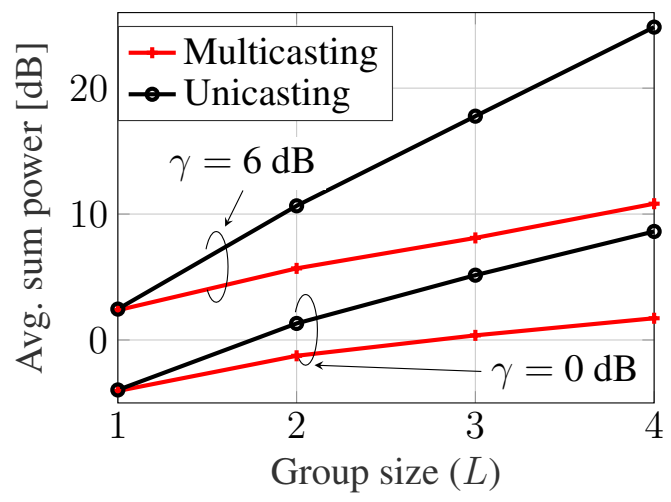

Fig. 4. Comparison between unicasting and multicasting transmissions with equal service levels and total resources.

provide good solutions to take the benefits from the antenna diversity. When the multicasting is applied in CRAN systems, the centralized baseband processing of CRAN can potentially lead to large delays since a significant part of the information processing is concentrated on the base band unit in the other end of the fronthaul link. To address this issue, caching of popular contents can provide significant benefits in terms of signaling overhead of the fronthaul/backhaul links and delays, by bringing the data closer to the end users [3], [4]. However, it imposes the same challenges, what information should be cached, where, how long and what is the proper size for the cache. To address these challenges, some solutions and discussions have been provided in [3], [4], [26].

\section{REFERENCES}

[1] "Ericsson, Ericsson Mobility Report, Nov. 2016." [Online]. Available: https://www.ericsson.com/assets/local/mobilityreport/documents/2016/ ericsson-mobility-report-november-2016.pdf

[2] "Ericsson, Evolving LTE to fit the 5G future, Jan. 2017." [Online]. Available: https://www.ericsson.com/publications/ericsson-technologyreview/archive/2017/evolving-lte-to-fit-the-5g-future

[3] M. Tao, E. Chen, H. Zhou, and W. Yu, "Content-centric sparse multicast beamforming for cache-enabled cloud RAN," IEEE Trans. Wireless Commun., vol. 15, no. 9, pp. 6118-6131, Sep 2016.

[4] D. Christopoulos, S. Chatzinotas, and B. Ottersten, "Cellular-broadcast service convergence through caching for CoMP cloud RANs," in Proc. IEEE Symp. Commun. Veh. Tech. in the Benelux (SCVT), Nov 2015, pp. $1-6$.
[5] N. D. Sidiropoulos, T. N. Davidson, and L. Z.-Q., "Transmit beamforming for physical-layer multicasting," IEEE Trans. Signal Process., vol. 54, no. 6, pp. 2239-2251, Jun. 2006.

[6] E. Karipidis, N. Sidiropoulos, and Z.-Q. Luo, "Quality of service and max-min fair transmit beamforming to multiple cochannel multicast groups," IEEE Trans. Signal Process., vol. 56, no. 3, pp. 1268-1279, Mar. 2008.

[7] D. Christopoulos, S. Chatzinotas, and B. Ottersten, "Weighted fair multicast multigroup beamforming under per-antenna power constraints," IEEE Trans. Signal Process., vol. 62, no. 19, pp. 5132-5142, Oct. 2014.

[8] D. Christopoulos, S. Chatzinotas, and B.Ottersten, "Multicast multigroup precoding and user scheduling for frame-based satellite communications," IEEE Trans. Wireless Commun., vol. 14, no. 9, pp. 4695-4707, Sep 2015.

[9] Z. Xiang, M. Tao, and X. Wang, "Coordinated multicast beamforming in multicell networks," IEEE Trans. Wireless Commun., vol. 12, no. 1, pp. 12-21, Jan. 2013.

[10] N. Bornhorst, M. Pesavento, and A. Gershman, "Distributed beamforming for multi-group multicasting relay networks," IEEE Trans. Signal Process., vol. 60, no. 1, pp. 221-232, Jan. 2012.

[11] D. Christopoulos, S. Chatzinotas, and B. Ottersten, "Multicast multigroup beamforming under per-antenna power constraints," in Proc. IEEE Int. Conf. Commun., Sydney, Australia, 2014, pp. 4704-4710.

[12] D. Christopoulos, S. Chatzinotas, and B.Ottersten, "Sum rate maximizing multigroup multicast beamforming under per-antenna power constraints," in Proc. IEEE Global Commun. Conference, Austin, Texas, USA, 2014, pp. 3354-3359.

[13] S. He, Y. Huang, S. Jin, and L. Yang, "Energy efficient coordinated beamforming design in multi-cell multicast networks," IEEE Commun. Lett., vol. 19, no. 6, pp. 985-988, Jun. 2015.

[14] O. Mehanna, N. Sidiropoulos, and G. Giannakis, "Joint multicast beamforming and antenna selection," IEEE Trans. Signal Process., vol. 61, no. 10, pp. 2660-2674, May 2013.

[15] O. Tervo, L.-N. Tran, H. Pennanen, S. Chatzinotas, M. Juntti, and B. Ottersten, "Energy-efficient coordinated multi-cell multigroup multicast beamforming with antenna selection, accepted to IEEE Int. Conf. Commun. Workshop," May 2017. [Online]. Available: https://arxiv.org/abs/1702.05632

[16] "Alcatel-Lucent, "eMBMS for more efficient use of spectrum", Nov. 2011." [Online]. Available: https://insight.nokia.com/embmsmore-efficient-use-spectrum

[17] 3GPP Technical Report 23.246, "Technical Specification Group Services and System Aspects; Multimedia Broadcast/Multicast Service (MBMS); Architecture and functional description ," Dec. 2016. [Online]. Available: https://portal.3gpp.org/desktopmodules/Specifications/SpecificationDetai ls.aspx?specificationId=829

[18] "Qualcomm, LTE Broadcast," 2014. [Online]. Available: https://www.qualcomm.com/invention/technologies/lte/broadcast

[19] A. Wiesel, Y. Eldar, and S. Shamai, "Linear precoding via conic optimization for fixed MIMO receivers," IEEE Trans. Signal Process., vol. 54, no. 1, pp. 161-176, Jan. 2006.

[20] O. Tervo, L.-N. Tran, and M. Juntti, "Optimal energy-efficient transmit beamforming for multi-user MISO downlink," IEEE Trans. Signal Process., vol. 63, no. 20, pp. 5574-5588, Oct. 2015.

[21] K. Nguyen, L. Tran, O. Tervo, Q. Vu, and M. Juntti, "Achieving energy efficiency fairness in multicell MISO downlink," IEEE Commun. Lett., vol. 19, no. 8, pp. 1426-1429, Aug. 2015.

[22] H. Pennanen, D. Christopoulos, S. Chatzinotas, and B. Ottersten, "Distributed coordinated beamforming for multi-cell multigroup multicast systems," in Proc. IEEE Int. Conf. Commun., May 2016, pp. 1-7.

[23] G. Venkatraman, A. Tölli, M. Juntti, and L. N. Tran, "Multi-group multicast beamformer design for MIMO-OFDM transmission," in Proc. European Wireless Conference, May 2016, pp. 1-6.

[24] A. Beck, A. Ben-Tal, and L. Tetruashvili, "A sequential parametric convex approximation method with applications to nonconvex truss topology design problems," Journal of Global Optimization, vol. 47, no. 1, pp. 29-51, 2010.

[25] S. Schaible, "Fractional Programming. I, Duality," Management Science, vol. 22 , no. 8, pp. 858-867, 1976.

[26] K. Poularakis, G. Iosifidis, V. Sourlas, and L. Tassiulas, "Exploiting caching and multicast for $5 \mathrm{G}$ wireless networks," IEEE Trans. Wireless Commun., vol. 15, no. 4, pp. 2995-3007, April 2016. 